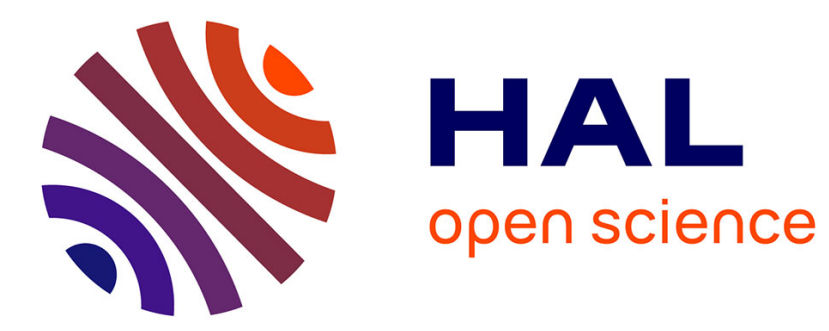

\title{
Path integral studies of positrons and positronium in fluids
}

\author{
B. Miller, Jie Chen, T. Reese, G. Worrell
}

\section{To cite this version:}

B. Miller, Jie Chen, T. Reese, G. Worrell. Path integral studies of positrons and positronium in fluids. Journal de Physique IV Proceedings, 1993, 03 (C4), pp.C4-31-C4-39. 10.1051/jp4:1993403 . jpa-00251446

\section{HAL Id: jpa-00251446 https://hal.science/jpa-00251446}

Submitted on 1 Jan 1993

HAL is a multi-disciplinary open access archive for the deposit and dissemination of scientific research documents, whether they are published or not. The documents may come from teaching and research institutions in France or abroad, or from public or private research centers.
L'archive ouverte pluridisciplinaire HAL, est destinée au dépôt et à la diffusion de documents scientifiques de niveau recherche, publiés ou non, émanant des établissements d'enseignement et de recherche français ou étrangers, des laboratoires publics ou privés. 


\title{
Path integral studies of positrons and positronium in fluids
}

\author{
B.N. MILLER, J. CHEN, T.L. REESE and G. WORRELL \\ Physics Department, Texas Christian University, Fort Worth, TX 76129, U.S.A.
}

\begin{abstract}
Experimental studies of positron and positronium decay in dense fluids suggest that these particles are capable of forming self-trapped states in some fluids in a broad region of temperature and density surrounding the liquid-vapor critical point. A central question in understanding the phenomena is whether the light particle (lp) actively creates a static potential well in the fluid in which it localizes, or randomly visits statistically favorable fluctuations. The fact that the experimental measurements yield a single, well defined, decay rate for each decay mode suggest that the environment of the Ip is static. Earlier mean field theories could not shed much light on this question. However, two recent applications of Path Integral Monte Carlo show that substantial fluctuations occur in the lp environment. In this report, the two applications of path integration to lifetime studies are reviewed. The quantitative distribution of fluctuations in the decay rate for each particle (positron and positronium atom) is presented. It is shown that the apparent conflict between theory and experiment is resolved for positronium (but not positron) decay when the time scale for the relaxation of fluctuations in the environment is taken into account. Finally, recent results concerning the ability of the analytic reference interaction site model (RISM) polaron theory to reproduce the exact PIMC calculations are described.
\end{abstract}

\section{Introduction}

The basic assumption of nearly all theories of positron and positronium decay in fluids is that the atomic and molecular translational degrees of freedom of the fluid obey classical mechanics, whereas the light particle (positron or positronium atom) obeys quantum mechanics.[1,2] To obtain an analytically tractable model for the important properties of the hybrid system, theorists have been forced to radicalize the adiabatic model, as it is known, with additional, far more drastic, assumptions.[3] This is readily seen with density functional type theories, (DFT's) which characterize the system by a single optimal wavefunction and average, local, fluid density, thus ignoring both quantum and classical fluctuations entirely.[4-6] It occurs to a lesser degree in the polaron type models which are based on a combination of statistical perturbation theory and mean field theory.[7] In the limit of low temperatures (in practice, on the order of $10 \mathrm{~K}$ ), it is expected that the quantum ground state will dominate, setting the stage for some version of DFT. However, at higher temperatures, one can neither expect that the light particle (lp) is represented by a single quantum state, nor that the lp-atom interaction is weak. As a consequence, predictions based on these analytical models, ingenious as they are, cannot be taken as serious representations of the adiabatic assumption, but rather as qualitative characterizations which approximately 
mimic some features of the source.

Unlikely as it seems, the actual test of an analytical model is not its ability to represent nature, but rather, how well it reproduces the parent adiabatic model. Of equal, or greater, importance is the ability of the adiabatic model to predict experimental measurements of the annihilation rate and the momentum distribution of the annihilation photons, and our ability to directly study its properties. Because the quantum particle has an infinite number of degrees of freedom, this is not a simple matter. At present, two approaches are available; Path Integral Monte Carlo (PIMC) and Quantum Molecular Dynamics (QMD). Each method requires prodigious computing power to implement. In addition to requiring long runs on large machines, QMD always requires some sort of mode truncation and regularization of the interaction.[8] In contrast, with present algorithms, PIMC can be checked for stability and convergence without forgoing complete control of the interactions. [9]

We started investigating path integral methods at T. C. U. in 1990 and presented preliminary computations at PPC3.[10] Since then, we have made substantial progress. We first developed analytic methods for expressing the annihilation rate and momentum distribution in terms of the path integral.[3] We then applied the method to a study of a thermalized positron in Xenon at $340 \mathrm{~K}$, where experimental data indicates that the $\mathrm{e}^{+}$annihilation rate is anomalously large at low to moderate density.[11] More recently we have applied the method to the Ps-Xenon system [12] and investigated the distribution of fluctuations for both $\mathrm{e}^{+}$and Ps.[13] At the present time we are studying the applicability of the analytic RISM-polaron theory to represent both the fluid and particle properties.[14]

In Section II we will describe the essential features of the discretized path integral and its application to both $\mathrm{e}^{+}$and Ps annihilation. In III the results of the studies of thermalized $\mathrm{e}^{+}$and Ps in Xenon will be summarized. In IV the distribution of fluctuations will be discussed for each system and the time scales for their relaxation will be estimated. In V, the results of the application of the RISM-polaron theory to each system will be compared with the PIMC simulations. The new information provided by PIMC and its relative merits for future investigations will be evaluated in VI.

\section{The Discretized Path Integral}

In the adiabatic model the atoms (or molecules) are classical objects and the lp (positron or positronium atom) obeys quantum mechanics. Represent the total inter-atomic interaction potential by $\mathrm{U}(\mathbf{R})$, and the total lp-atom interaction by $W(\mathbf{r}, \mathbf{R})$, where $\mathbf{R}$ represents the set of atomic (or molecular) positions $\left(\mathbf{R}_{\mathbf{j}}\right\}$ and $\mathbf{r}$ denotes the position operator of the $l p$ (positron or positronium center of mass). Then the Hamiltonian of the quantum particle is

$$
H=-\left(\hbar^{2} / 2 m\right) \Delta+W(\mathbf{r}, \mathbf{R})
$$

and the statistical average of the quantum operator $O(\mathbf{r}, \mathbf{R})$ is given by

$$
\langle O(\mathbf{r}, \mathbf{R})\rangle=(1 / Z) \int \mathrm{d} \mathbf{R} \exp (-\beta U) \int \mathrm{d} \mathbf{r}\langle\mathbf{r}|O(\mathbf{r}, \mathbf{R}) \exp (-\beta \mathrm{H})| \mathbf{r}\rangle,
$$

where $\mathrm{Z}$ is the configurational partition function, $\mathrm{d} \mathrm{d} r$ represents the usual trace over the quantum states of $H$ in the position representation and, as usual, $\beta=1 / k T$.[15] Typically $U$ and $W$ are assumed to be pairwise additive,

$$
U(\mathbf{R})=\Sigma_{1 \leq i<j \leq N} u\left(R_{i}-R_{j}\right), \quad w(r, R)=\Sigma_{1 \leq j \leq N} w\left(r-R_{j}\right),
$$

e.g. $u$ may be a Lennard-Jones $(12,6)$ potential while the form of $w$ will depend on whether the lp is a 
positron or positronium atom. [1]

For the purposes of computing the statistical average, Feynman proved, by inserting $\mathrm{p}-1$ intermediate positions in (2), that a single quantum particle can be represented by a classical harmonic chain consisting of $p$ pseudo-particles.$[15,16]$ In this representation,

$$
\begin{aligned}
& \int \mathrm{d} \mathbf{r}\langle\mathbf{r}|O(\mathbf{r}, \mathbf{R}) \exp (-\beta H)| \mathbf{r}\rangle \rightarrow \int \mathrm{d} \mathbf{r} O\left(\mathbf{r}_{1}, \mathbf{B}\right) \exp [-\beta \Phi(\mathbf{r}, \mathbf{R})], \\
& \Phi(\mathbf{r}, \mathbf{R}) \equiv \sum_{1<\alpha<\mathrm{p}}\left[\left(2 \mathrm{mp} / \beta^{2} \hbar^{2}\right)\left|\mathbf{r}_{\alpha+1}-\mathbf{r}_{\alpha}\right|^{2}+(1 / \mathrm{p}) \mathrm{W}\left(\mathbf{r}_{\alpha}, \mathbf{R}\right)\right],
\end{aligned}
$$

where $\boldsymbol{c}$ represents the set of positions $\left\{\mathbf{r}_{\alpha}\right\}$ of the pseudo-particles and $\mathbf{r}_{p+1}=\mathbf{r}_{1}=\mathbf{r}$ (the chain is closed). Thus each pseudo-particle (pp) interacts with the fluid atoms via the reduced potential $\mathrm{W} / \mathrm{p}$ and with its nearest neighbors via a harmonic potential with effective temperature dependent force constant $4 \mathrm{mp} / \beta^{2} \hbar^{2}$. The equivalence is exact in the limit $\mathrm{p} \rightarrow \infty$. The problem of computing the equilibrium properties of the system is consequently reduced to sampling the scalar (classical) distribution $\exp [-\beta(U+\Phi)]$ in the configuration space of atomic and pp coordinates $(\mathbf{R}, \mathbf{r})$. Specific algorithms have been developed to accomplish this task and go under the name of path-integral Monte Carlo (PIMC).[17, 18]

Two structural properties of fundamental importance are the rms separation between a pair of pp's separated by $s$ harmonic bonds, $\mathrm{D}_{\mathrm{s}}$, and the $\mathrm{lp}$-atom radial distribution function, $\mathrm{g}_{\mathrm{fp}}(\mathrm{r})$ :

$$
\mathrm{D}_{\mathrm{s}}^{2}=(1 / \mathrm{p}) \Sigma_{\alpha}\left\langle\left|\mathbf{r}_{\alpha+\mathrm{s}^{-}} \mathbf{r}_{\alpha}\right|^{2}\right\rangle, \quad \rho \mathrm{g}_{\mathrm{fp}}(\mathbf{r})=(1 / \mathrm{p}) \Sigma_{\alpha, j}\left\langle\delta\left(\mathbf{r}_{\alpha}-\mathbf{R}_{\mathrm{j}}-\mathbf{r}\right)\right\rangle
$$

Here the angle brackets, which do not refer to a specific quantum state, imply the complete statistical ensemble average, and $\rho$ is the mean fluid density. In the absence of the lp-atom interaction (i.e. if $W=0$ ), $g_{f p}$ is unity and $p^{2} D_{s}{ }^{2}=3 \Lambda_{l p}{ }^{2} s(p-s)$, where $\Lambda_{l p}=\sqrt{ }\left(2 \pi \beta \hbar^{2} / m\right)$ is the thermal wavelength of the Ip. Clumping of the chain is indicated by strong reductions in $D_{s}$ at the midpoint of the chain $(s=p / 2)$ below the free particle value $\left(\Lambda_{\mathrm{lp}} \sqrt{ }(3 / 4)\right)$ and is attributed to the dominance of localized lp states, while deviations of $g_{f p}$ from unity describe changes the average fluid environment in the neighborhood of the lp induced by its presence.[7]

The average annihilation rate of the positron is proportional to the overlap between the positron wavefunction and the atomic electrons. It is given by [3]

$$
\langle\lambda\rangle=\rho \int d r f(r) g_{f p}(r)
$$

in appropriate units. For the pick-off decay rate of ortho-positronium (7) must be altered to account for the penetration of the positron into the electron cloud of a fluid atom. This is accomplished by first convolving $f$ with the square of the ground state positronium wavefunction.[12] We note in passing that the momentum distribution of the annihilating photons can also be expressed in terms of a path integral, but here the complete off-diagonal density matrix is required and the chain is no longer closed.[3]

\section{Application to $\mathrm{e}^{+}$and Ps in Xenon}

Our first major application of the PIMC was to the $\mathrm{e}^{+}$-Xenon system.[11] Generic plots of the $\mathrm{e}^{+}$ decay rate versus density on rare gas isotherms show linear behavior in the limit of low and high densities 
with the usual "bump" in the critical region suggesting the existence of localized states. [19] Xenon was chosen because experimental measurements of the annihilation rate exhibited an anomalously large, nonlinear, increase with density on two isotherms $(300 \mathrm{~K}$ and $340 \mathrm{~K})$ above the critical temperature (289K).[20]

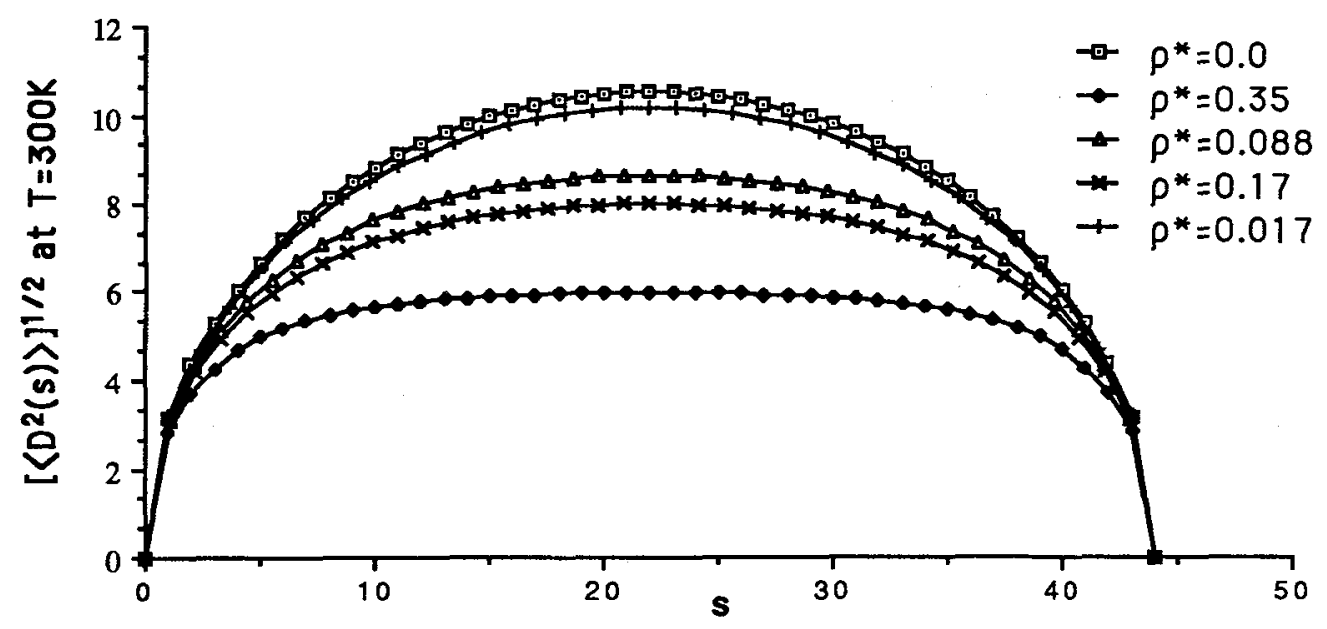

Fig. 1. Root mean square displacement between polymer particles separated by s-1 intervening particles. The system is Positronium in Xenon at $300 \mathrm{~K}$. Note the increasing polymer compression with increasing density, demonstrating that the Ps atom is becoming more localized.

A Lennard-Jones $(12,6)$ potential was chosen for $\mathrm{u}$ and an effective $\mathrm{e}^{+}$-atom interaction potential $\mathbf{w}(\mathbf{r}-\mathbf{R})$ was constructed by smoothing the sharp cut-off in the potential Nakanishi and Schrader and produced from fits to $\mathrm{e}^{+}-\mathrm{Xe}$ scattering data.[21] In units of length where the Lennard-Jones length parameter $\sigma=1$, simulations were carried out at $\rho=0.01,0.05,0.15$, and 0.5 (in these units the critical density is about 0.35 ) at $\mathrm{T}=340 \mathrm{~K}$. The simulations showed that the positron was highly localized (small $\mathrm{D}_{\mathrm{S}}$ ) in a large cluster (strongly peaked $\mathrm{g}_{\mathrm{fp}}$ ), or droplet, consisting of about 35 Xenon atoms, at the two intermediate densities (and, to within a constant factor, reproduced the shape of the experimental plot of $\langle\lambda\rangle$ versus $p .[11]$ It was surprising that the positron was less localized at the highest density considered, and that the strongest localization occurred well below the critical density.

We also decided to study Ps in Xenon because, of all the rare gases studied experimentally, it has the highest critical temperature. The smaller thermal wavelength helped control the system size in the simulations, which aided convergence. In contrast with $\mathrm{e}^{+}$, analytic potentials have not been developed for the Ps-Xe interaction potential, and Ps-Xe scattering data is not available. Since the Fermionic repulsion of the electron in Ps dominates the interaction, we simply used a hard sphere potential with a radius of $a_{0}+\sigma / 2$, corresponding to the geometric separation of the two atoms.[12] Simulations were carried out on two isotherms $(T=300 \mathrm{~K}$, and $\mathrm{T}=340 \mathrm{~K})$ at scaled densities $\rho=0.017,0.088,0.17$ and 0.35 (the critical density). The infinitely repulsive interaction caused a very low acceptance rate in the simulations and forced us to develop a new sampling algorithm. $[12,22]$ 


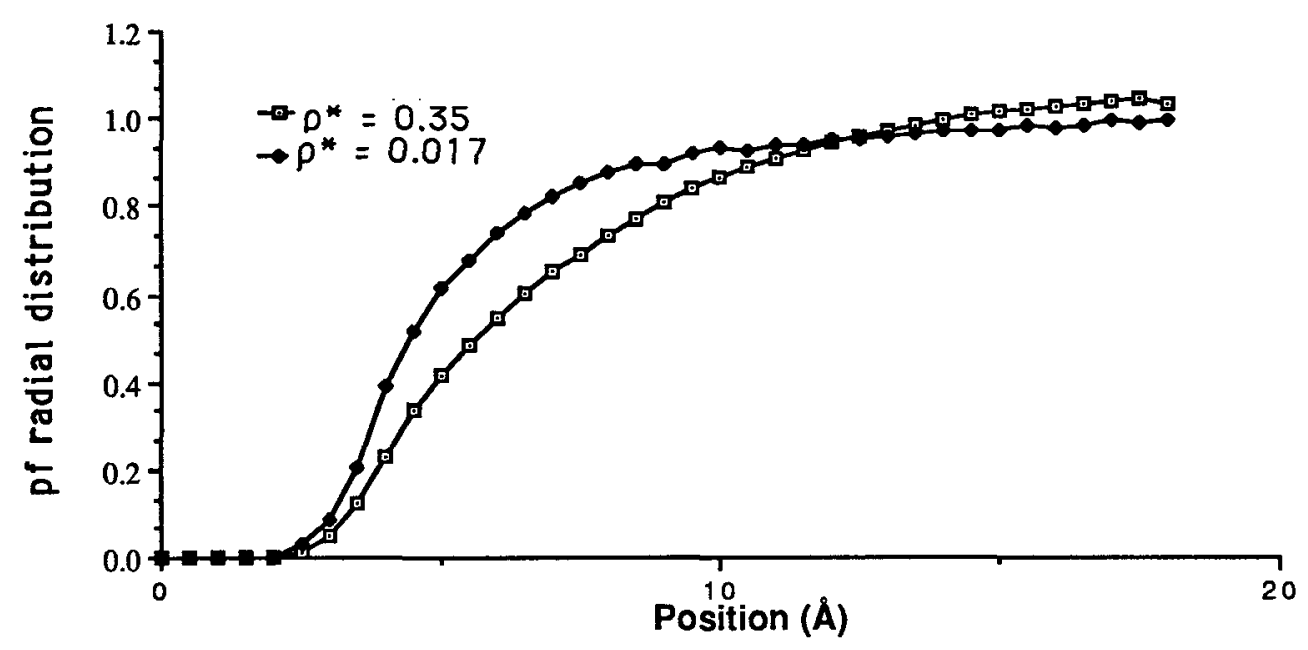

Fig. 2. Polymer-fluid atom radial distribution function for positronium in Xenon at $300 \mathrm{~K}$. Note that the fluid displsacement is greater at the critical density (0.35).

Consistent with the experimental data, the model predictions here were much less dramatic than for $\mathrm{e}^{+}$. As determined from studies of $\mathrm{D}_{\mathrm{s}}$ and $\mathrm{g}_{\mathrm{fp}}$ (Figs. 1 and 2), the degree of localization, and the size of the cavity carved out by the positronium atom gradually increased with density on each isotherm. The effect was slightly stronger at the lower temperature. While the experimental plots of the pick-off decay rate versus density show a characteristically flat plateau in the critical region, the predicted reduction in slope was smaller (Fig. 3).[23]

\section{Analysis of Fluctuations}

In addition to determining the average annihilation rates at a specified density and temperature, PIMC was used to simultaneously generate the complete decay rate equilibrium distribution. [13] This is not possible with the earlier models since they are mean field theories and, for the first time, provides the opportunity to answer questions concerning the constancy of the lp's environment. This information is quantified by $\zeta_{\lambda}=\sigma_{\lambda} /\langle\lambda\rangle$, where $\sigma_{\lambda}{ }^{2}$ is the variance of the decay rate calculated for each distribution (Fig. 4). For o-Ps in Xenon at $340 \mathrm{~K}, 0.83>\zeta_{\lambda}>0.44$ for $0.017<\rho<0.35$ [12]. For $\mathrm{e}^{+}$in Xenon at $\mathrm{T}=340 \mathrm{~K}$, $1.2>\zeta_{\lambda}>0.18$ for $0.01<\rho<0.5$ [11]. Thus the simulations clearly demonstrate that, in each case, the local environment of the lp fluctuates strongly. The question that needs to be addressed here is how these large variations in decay rate can be reconciled with the experimental results which yield a single, easily resolved, annihilation rate.

The quantity that is actually measured in the laboratory is the mean value of $P(t)$, the probability of survival at time $t$ [24]. Since the annihilation process is Markovian, for a given positron (or positronium atom)

$$
P(t)=\exp \left[-\int_{0}^{t} \lambda\left(t^{\prime}\right) d t^{\prime}\right]
$$

where, here, $\lambda(t)$ is the instantaneous annihilation rate experienced by the unstable particle. To determine under what conditions an experiment should yield a single value for $\lambda$, let $\langle\lambda\rangle$ represent the mean 
annihilation rate as above and let $\delta \lambda(t)$ denote it's fluctuation at time $t$. Then, through second order in $\delta \lambda$,

$$
\ln \langle P(t)\rangle=-\langle\lambda\rangle t+\langle\lambda\rangle^{2} \zeta \lambda^{2} \int_{0}^{t} d \tau c(\tau)(t-|\tau|)
$$

where $c(\tau)=\langle\delta \lambda(\tau) \delta \lambda(0)\rangle /\left\langle\delta \lambda(0)^{2}\right\rangle$ is the decay rate time-correlation function normalized to unity at $\tau=0$.

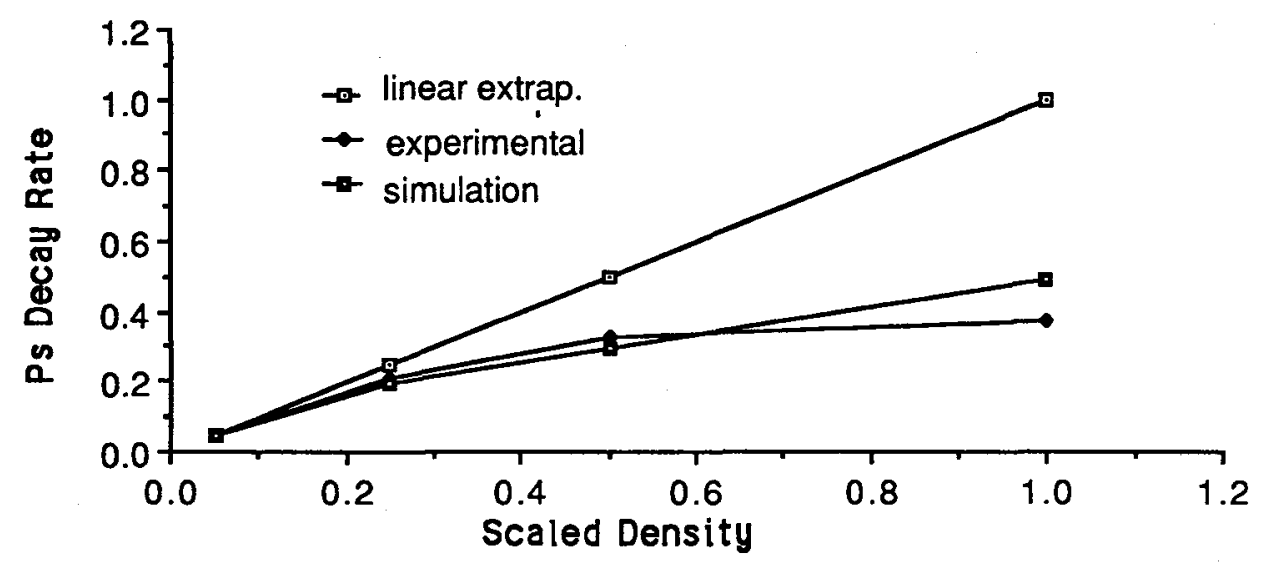

Fig. 3. Positronium decay rate versus density in Xenon at $300 \mathrm{~K}$.

If the second term on the right of (9) is sufficiently small, the experiment will detect the average. This can occur if (a) $\zeta_{\lambda}$ is very small, or (b) $c(\tau)$ decays on a time scale $\tau_{\lambda}$ much shorter than $t \approx 1 /(\lambda\rangle$, the time of observation. However, we have already demonstrated from PIMC that $\zeta_{\lambda}$ is of order unity so (a) doesn't apply and we consider (b).

Because $\lambda(t)$ depends on the atomic positions $\left\{\mathbf{R}_{\mathbf{j}}(t)\right\}$, we assume that its evolution is slaved to the evolution of density fluctuations in the fluid. For simplicity let's assume that the relaxation time $\tau_{\lambda}$ is essentially that of the density fluctuation which it has stabilized. For both $\mathrm{e}^{+}$and o-Ps, PIMC indicates that the diameter of the disturbed region in the fluid, for which $\mathrm{g}_{\mathrm{fp}}$ is different than unity, is on the order of $20 \AA$. In the case of $\mathrm{e}^{+}$, a dense droplet is created in its vicinity [11]. Thus a good estimate of $\tau_{\lambda}$ for the positron should be the time required for an atom to diffuse a distance of $20 \AA$ in the dense fluid. In contrast, a cavity is created around o-Ps which is nearly vacant [12]. An estimate of the relaxation time of this structure is approximately the time for an atom to ballistically traverse the cavity. Consequently the relaxation time for o-Ps is expected to be much less than that for $\mathrm{e}^{+}$.

Experimental measurements [25] indicate that the diffusion constant of Xenon varies slowly with the thermodynamic parameters in a region above the critical density and temperature with a value of approximately $D \approx 2.0 \times 10^{-8} \mathrm{~m}^{2} \mathrm{~s}^{-1}$, which yields $\tau_{\lambda} \approx(20 \AA)^{2} / \mathrm{D} \approx 0.2 \mathrm{~ns}$. Now typical values of $\langle\lambda\rangle \mathrm{t} \approx 1$ for $\mathrm{e}^{+}$annihilation in $\mathrm{Xe}$ at $300 \mathrm{~K}$ range from about 0.7 to $2.0 \mathrm{~ns}$, yielding $0.1<\tau_{\lambda} / \mathrm{t}<0.25$. For the case of positronium, $1 /\langle\lambda\rangle \approx t$ is of much longer duration, since the vacuum lifetime of o-Ps is about $140 \mathrm{~ns}$. In addition, the relaxation time is controlled by ballistic motion, which is much more rapid than diffusion. Consequently, $\tau_{\lambda} / t \ll 1$ for 0 -Ps decay. 


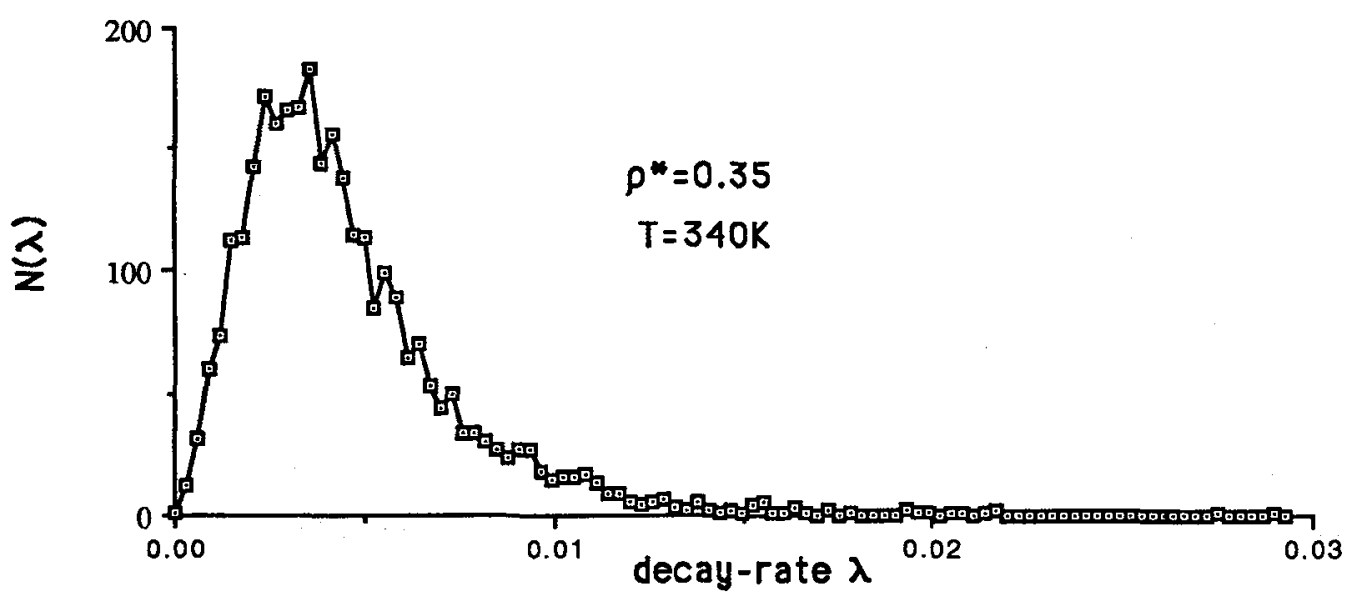

Fig. 4. Distribution of fluctuating decay rate from simulation.

\section{RISM-polaron Theory}

Most analytical theories of self-trapping in fluids have been based on some form of density functional theory. Their product is a unique lp wavefunction and local fluid density which, together, extremize a thermodynamic potential. Because they are mean field theories, all of these solutions ignore fluctuations and have the annoying property of suddenly disappearing in certain ranges of density and temperature of the host fluid. Thus, they hold out no hope for modeling the lp either at low or high densities, or in the transition region between trapped and extended states, where fluctuations play a dominant role.

The only exception to the above is provided by the RISM-polaron theory developed by Chandler et. al. $[7,26]$ Rather than being derived from DFT, it is based on the path integral and provides a statistical distribution of the system properties. RISM theory (reference interaction site model) was originally introduced by Chandler to study the structure of molecular fluids. Thus it was a natural choice for modeling the "polymer" which arises from the discretized path integral. There is not room here to give the mathematical description of the theory, but it is only important to realize that, unlike DFT's, its products are $g_{f p}$ and $D_{s}$, so it holds out the potential for revealing the degree of localization and the annihilation rate of $\mathrm{e}^{+}$or Ps over a wide range of circumstances. The theory results in coupled equations for $\mathrm{g}_{\mathrm{fp}}$ and $\omega(\mathbf{r})$ (the polymer distribution function) which are solved iteratively. While complex in nature, convergence is obtained much more rapidly than with PIMC, and can be carried out on a workstation.

As a test of the model, we have just completed applying RISM-polaron theory to the case of $\mathrm{e}^{+}$in Xenon.[14] We used the same interaction potential as in our PIMC studies so we were able to directly compare the predictions with numerically accurate simulations at four different densities. We were also able to carry out the analytical work at many other densities and temperatures. We found that the qualitative features of the predictions agree well with PIMC. However, there were some important differences: (a) The peak of $\mathrm{g}_{\mathrm{fp}}$ near the potential minimum was weaker and broader; (b) The overall shape of the plot of $\langle\lambda\rangle$ was similar to that of PIMC, and the experimental data, but noticeably smaller at each value of the density. It is interesting that the RISM theory predicts a local minimum in the decay rate at about $p=0.5$. The experimental data of Tuomisaari et. al. suggest that such a minimum may occur, but there are insufficient data values to resolve the issue.[20] Since there are even fewer PIMC generated values of $\lambda$, this issue can't be resolved here either. 


\section{Conclusions}

The expense of carrying out path integral Monte Carlo calculations has engendered criticism. However, it is abundantly clear from the above that PIMC provides a vital test, and in some ways the only test, of other, less complete, theoretical models. Moreover PIMC is, to date, the most effective and accurate means for calculating the equilibrium properties of the adiabatic lp-fluid system. In general, it has resulted in a clearer understanding of localization. For example, we now recognize that the degree of localization, quantified by the reduction of $D_{s}$ from the free particle value, is only indirectly related to the lp-atom correlation function $g_{f p}[26]$ which, in tum, should not be confused with the local density profile produced by DFT. [11, 12] Angular correlation experiments are controlled by $D_{S}$, whereas lifetime measurements are determined by $\mathbf{g}_{\mathrm{fp}}$.

The origin of the very large clusters revealed in the $\mathrm{e}^{+}$-Xenon system are non-intuitive and difficult to comprehend. They are a result of the deep minimum in the $\mathrm{e}^{+}-\mathrm{Xe}$ interaction potential produced by the large polarizability of atomic Xenon. Recent ab-initio studies of the $\mathrm{e}^{+}-\mathrm{Xe}$ system demonstrates that there is a virtual resonance in the interaction potential near zero energy, which may contribute to the strong peak in $\mathrm{g}_{\mathrm{fp}}$. . 27] This should be kept in mind when considering DFT type models which typically replace the actual interaction potential with a mathematically more tractable contact potential.[28] PIMC strongly suggests that the details of the potential strongly affect the lifetime predictions because they directly influence the short range behavior of $g_{f p}$ which, along with the electron density, determines the mean decay rate. The same could be said for the Ps-Xenon studies, where the choice of the hard sphere interaction [12] failed to produce the degree of localization suggested by the laboratory measurements of the pick-off decay rate.[13]

The very large fluctuations which occur in each trial system could not be predicted by a mean field theory. Nor could they be experimentally resolved. The estimated relaxation time for atomic clusters surrounding a positron suggest that it may be possible to experimentally resolve, or at least detect, the fluctuating distribution of positron lifetimes. In contrast, the "back of the envelope" estimates given here suggest that fluctuations within a Ps "bubble" are too rapid to be experimentally detected.

Of all of the analytical theories considered to date, the RISM-polaron theory holds out the most promise. We have shown that it replicates the essential features of the PIMC studies of $\mathrm{e}^{+}-\mathrm{Xenon}$ system over the complete range of densities, but underestimates their strength. It will be interesting to see if improvements in this theory are forthcoming.

\section{Acknowledgements}

The authors are grateful for the support of the Welch Foundation, the Pittsburgh Supercomputing Center, and the Research Foundation of Texas Christian University.

\section{References}

1. I. T. Iakubov and A. G. Khrapak, Prog. Phys. 45, 697 (1982).

2. J. Hernandez, Rev. Mod. Phys. 63, 675, (1991).

3. B. N. Miller and Y. Fan, Phys. Rev. A 42, 2228 (1990).

4. M. J. Stott and E. Zaremba, Phys. Rev. Lett. 38, 1493 (1977);

R. M. Nieminen, M. Manninen, I. Valimaa, and P. Hautojarvi, Phys. Rev. A 21, 1677 (1980);

C. Ebner and C. Punyanita, Phys. Rev. A 19, 856 (1979).

5. B. N. Miller and T. L. Reese, Phys. Rev. A 39, 4735 (1989).

6. T. Reese and B. N. Miller, Phys. Rev. A 42, 6068 (1990).

7. A. L. Nichols III, D. Chandler, Y. Singh and D. M. Richardson,

J. Chem. Phys. 81, 5109 (1984). 
8. R. Kalia, P. Vashishta, and S. W. deleeuw, J. Chem. Phys. 90, 6802 (1989).

9. D. F. Coker, B. J. Berne and D. Thirumalai, J. Chem. Phys. 86, 5689 (1987).

10. Bruce N. Miller, Proceedings of the Third International Workshop on Positron and Positronium

Chemistry, ed. Y. C. Jean, (World Scientific, Singapore, 1990), p. 274.

11. G. A. Worrell and Bruce N. Miller, Phys. Rev. A 46, 3380 (1992).

12. T. Reese and B. N. Miller, Phys. Rev. E, 47, 2581 (1993).

13. B. N. Miller, T. Reese, and G. Worrell, Phys. Rev. E 47, (1993).

14. J. Chen and B. N. Miller, submitted to Phys. Rev. E

15. R. P. Feynman, Statistical Mechanics (Benjamin; Reading, Mass.; 1979).

16. R. P. Feynman and A. R. Hibbs, Quantum Mechanics and Path Integrals (McGraw-Hill, New York, 1965).

17. E. L. Pollock and D. M. Ceperley, Phys. Rev. B 30, 2555 (1984);

D. M. Ceperley and E. L. Pollock, Phys. Rev. Lett. 56, 351 (1986).

18. M. Sprik, M. L. Klein and D. Chandler, Phys. Rev. B 31, 4234 (1985); Phys. Rev. B 32, 545 (1984).

19. M. Tuomisaari, K. Rytsola, and P. Hautojarvi in Positron Annihilation Studies of Fluids, ed. S. Sharma (World Scientific, Singapore, 1988), p. 444 .

20. M. Tuomisaari and K. Rytsola, ref. 19, p. 77.

21. H. Nakanishi and D. Schrader, Phys. Rev. 34, 1810 (1986).

22. T. Reese and B. N. Miller, submitted to J. Comp. Phys.

23. M. Tuomisaari, K. Rytsola, and P. Hautojarvi, Phys. Lett. 112A, 279 (1985);

M. Tuomisaari, K. Rytsola, R. M. Nieminen, and P. Hautojarvi, J. Phys. B 19, 2667 (1986).

24. J.K. Percus, ref. 19, p. 96.

25. P. W. E. Peereboom, H. Luigjes, and K. O. Prins, Physica A 156, 260 (1989).

26. Y. Fan and B. N. Miller, J. Chem. Phys. 93, 4322 (1990).

27. V. A. Dzuba, V. V. Flambaum, W. A. King, B. N. Miller, and O. P. Sushkov, Physica Scripta, in press.

28. X. Z. Yan and S. T. Tsai, Phys. Rev. B 46, 4704 (1992). 